\section{T Lymphocytes Attenuate Dermal Scarring by Regulating Inflammation, Neovascularization, and Extracellular Matrix Remodeling}

Xinyi Wang, ${ }^{1}$ Swathi Balaji, ${ }^{1}$ Emily H. Steen, ${ }^{1}$ Hui Li, ${ }^{1}$ Meredith M. Rae, Alexander J. Blum, ${ }^{1}$ Qi Miao, ${ }^{1}$ Manish J. Butte, ${ }^{2,3}$ Paul L. Bollyky, ${ }^{4}$ and Sundeep G. Keswani, ${ }^{1, *}$

${ }^{1}$ Laboratory for Regenerative Tissue Repair, Division of Pediatric Surgery, Department of Surgery, Texas Children's Hospital and Baylor College of Medicine, Houston, Texas.

${ }^{2}$ Division of Immunology, Allergy, and Rheumatology, Department of Pediatrics, University of California, Los Angeles, Los Angeles, California.

${ }^{3}$ Department of Microbiology, Immunology, and Molecular Genetics, University of California, Los Angeles, Los Angeles, California.

${ }^{4}$ Division of Infectious Diseases, Department of Medicine, Stanford University School of Medicine, Stanford, California.

Objective: While tissue injury and repair are known to involve adaptive immunity, the profile of lymphocytes involved and their contribution to dermal scarring remain unclear. We hypothesized that restoration of $\mathrm{T}$ cell deficiency attenuates dermal scarring.

Approach: We assessed the temporal-spatial distribution of $\mathrm{T}$ lymphocytes and their subtypes during the physiological dermal wound repair process in mice. Also, we compared the scarring outcomes between wild-type (WT) and severe combined immunodeficient (SCID) mice, which are lymphocyte deficient. Complementary gain-of-function experiments were performed by adoptively transferring lymphocyte subsets to validate their contribution to tissue repair in wounded SCID mice. Results: $\mathrm{CD}^{+} \mathrm{T}$ lymphocytes were present within dermal wounds of WT mice beginning on day 1 and remained through day 30. Wounds of SCID mice exhibited accelerated closure, increased inflammation, limited neovascularization, and exacerbated scarring compared with WT mice. Conversely, transfer of either mixed B and $\mathrm{T}$ lymphocytes or $\mathrm{CD} 4^{+}$lymphocytes alone into SCID mice resulted in moderated healing with less inflammation, collagen deposition, and scarring than control SCID wounds. In contrast, transfer of other lymphocyte subsets, including helper $\mathrm{T}$ lymphocytes $\left(\mathrm{CD}^{+} \mathrm{CD}^{+} \mathrm{CD} 25^{-}\right), \mathrm{CD} 8^{+} \mathrm{T}$ cells and $\mathrm{B}$ cells, or regulatory $\mathrm{T}$ lymphocytes $\left(\mathrm{CD} 4^{+} \mathrm{CD} 25^{+} \mathrm{CD} 127^{\text {low }}\right)$, did not reduce scar.

Innovation: The finding that lymphocytes delay wound healing but reduce scar is novel and provides new insights into how dermal scarring is regulated.

Conclusion: Our data support a suppressive role for $\mathrm{CD} 4^{+} \mathrm{T}$ cells against inflammation and collagen deposition, with protective effects in early-stage dermal wound healing. These data implicate adaptive immunity in the regulation of scarring phenotypes.

Keywords: wound healing, extracellular matrix, adaptive immunity, lymphocytes, inflammation

(c) Xinyi Wang et al., 2019; Published by Mary Ann Liebert, Inc. This Open Access article is distributed under the terms of the Creative Commons Attribution Noncommercial License (http://creativecommons .org/licenses/by-nc/4.0/) which permits any noncommercial use, distribution, and reproduction in any medium, provided the original author(s) and the source are cited.
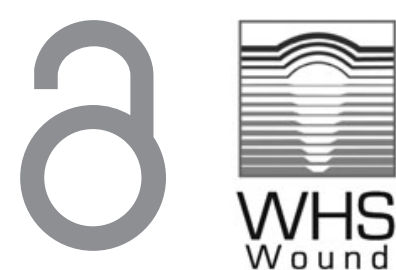

Wound

Healing

Society

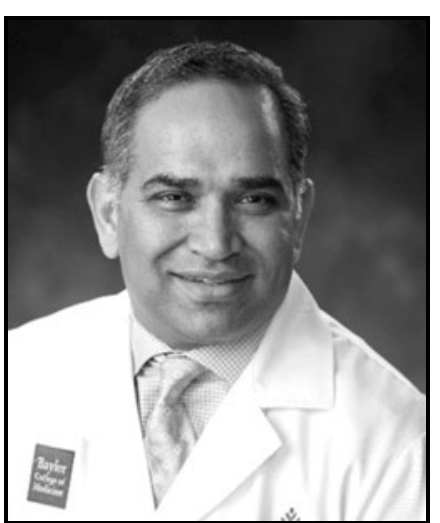

Sundeep G. Keswani, MD, FACS, FAAP

Submitted for publication March 7, 2019. Accepted in revised form May 23, 2019.

*Correspondence: Laboratory for Regenerative Tissue Repair, Division of Pediatric Surgery, Department of Surgery, Texas Children's Hospital and Baylor College of Medicine, Feigin Center, C.450.06, 1102 Bates Avenue, Houston, TX 77030 (e-mail: keswani@bcm.edu). 


\section{INTRODUCTION}

SimilaR Tо MOsт organs, repair of dermal tissues involves inflammatory reactions and recruitment of cell mediators of diverse lineage origins, including immune cells. ${ }^{1,2}$ When skin is injured, it undergoes a complex sequence of repair events, which include platelet-mediated hemostasis, local lymphocyte activation with myeloid cell infiltration, skin cell proliferation, and extracellular matrix (ECM) remodeling, often resulting in scar formation. ${ }^{3,4}$ Notably, inflammation is an essential phase of wound repair, and either insufficient or exacerbated inflammatory responses can lead to excessive scar formation and reduced tissue integrity. However, the impact of specific lymphocyte subsets and the degree of their regulatory contribution to scarring in the skin and other tissues are not completely understood.

Lymphocytes comprise a multifaceted cell lineage of hematopoietic origin, which are known to carry out prominent immune functions. Lymphoid progenitor cells are differentiated into $\mathrm{B}$ and $\mathrm{T}$ lymphocytes that respectively account for humoral and cell-mediated adaptive immunity. Within the $\mathrm{T}$ lymphocyte lineage, $\mathrm{CD} 4^{+} \mathrm{T}$ cells induce other immune cells to release $\mathrm{T}$ cell cytokines and modulate immune responses, whereas $\mathrm{CD}^{+} \mathrm{T}$ cells are recognized by their cell-mediated toxicity. Among the helper T cell subsets, a distinct subgroup expressing the Forkhead Box P3 (FoxP3) gene are known as regulatory T cells (Treg cells). Treg cells suppress overactive immunity, enable tissue tolerance, and restore homeostasis. ${ }^{5,6}$

Several studies have examined the impact of lymphocyte subsets on wounds. For instance, transfer of mature B cells, but not $\mathrm{T}$ cells, accelerated diabetic wound healing and reduced scar formation. ${ }^{7}$ In contrast, the absence of $\mathrm{CD} 8^{+} \mathrm{T}$ cells in athymic nude mice correlated with reduced scar formation, ${ }^{8,9}$ whereas Wong et al. demonstrated that athymic nude mice lacking mature $\mathrm{T}$ cells had hypertrophic scarring. ${ }^{10,11}$ Together, these studies suggest that $B$ and $T$ cells may impact scarring phenotypes, ${ }^{12,13}$ but the contribution of specific lymphocyte subsets remain largely unclear.

To better define the functional contribution of $\mathrm{T}$ cells to dermal wounds, we examined the spatial distribution of $\mathrm{T}$ lymphocyte populations during dermal tissue repair in wild-type (WT) mice and performed loss- and gain-of-function experiments in severe combined immunodeficient (SCID) mice with wound healing assessments.

\section{CLINICAL PROBLEM ADDRESSED}

Dermal scarring poses a significant health and economic burden that goes beyond cosmetic impact, often resulting in significant physical and psycho- social morbidity. Despite extensive studies, the factors that govern dermal scar formation are not completely understood, and few options for clinically effective treatments exist. Thus, uncovering the mechanisms that determine the transition from inflammation to scarring could provide key clues for scar-reducing therapies. Notably, our data support the notion that lymphocytes play a significant role in the physiological scarring process and that $\mathrm{CD} 4^{+}$ $\mathrm{T}$ lymphocyte subsets may hold immunotherapy leverage for anti-scarring medicine.

\section{MATERIALS AND METHODS \\ Animal care}

C57BL/6J (WT) and B6.CB17-Prkdc ${ }^{\text {scid/SzJ (SCID) }}$ mice were purchased from Jackson Laboratory (Bar Harbor, ME), bred, and maintained under pathogenfree conditions with access to food and water $a d$ libitum in the Texas Children's Hospital Feigin Center animal facility. Protocols for animal use were approved by the Institutional Animal Care and Use Committee at Baylor College of Medicine.

\section{Lymphocytes isolation and flow cytometry}

Female C57BL/6J mice served as donors. Total lymphocytes were isolated from mouse spleens using established protocols. ${ }^{14}$ In brief, splenocytes were pooled from multiple donor mice and extracted by tissue friction, resuspended with $5 \mathrm{~mL}$ of RPMI (Corning, New York, NY), and filtered with a $70-\mu \mathrm{m}$ cell strainer (Falcon; Corning) to form a single-cell suspension. Cells were spun down, and after supernatant removal, they were resuspended in $1 \mathrm{~mL}$ of ACK lysis buffer (Gibco, New York, NY) for $5 \mathrm{~min}$ at room temperature to lyse the red blood cells. T lymphocyte subsets were sorted from the mouse splenocyte suspension using a CD $4^{+} \mathrm{CD} 25^{+}$ Regulatory T Cell Isolation Kit (Cat. No. 130-091041; Miltenyi Biotec, San Diego, CA) following the manufacturer's protocol. All purified lymphocyte populations were used for adoptive transfer experiments within $6 \mathrm{~h}$ of wounding and isolation.

We examined the engraftment 7 days after adoptive transfer. Cells were isolated from individually treated mouse spleens and stained with fluorescently labeled antibodies, specifically, antimouse CD3 (100324; Biolegend), anti-mouse CD4 (11-0041-82; Biolegend), anti-mouse CD8a (450081-80; eBioscience), anti-mouse CD25 (12-025182; eBioscience), anti-mouse CD127 (566300; BD Biosciences, San Jose, CA), and anti-mouse CD45R/B220 (552772; BD Biosciences). ${ }^{15}$ The following cell subsets were analyzed by a BD LSRII flow cytometer (BD Biosciences): $\mathrm{CD}^{+} \mathrm{T}$ and $\mathrm{B} 220^{+}$ B lymphocytes, $\mathrm{CD}^{-} \mathrm{T}$ cells $\left(\mathrm{CD}^{+} \mathrm{CD}^{-} \mathrm{a}^{+}\right.$or 
$\mathrm{CD}_{22}{ }^{+}$, after $\mathrm{CD} 4^{+} \mathrm{T}$ cell depletion), $\mathrm{CD} 4^{+} \mathrm{CD} 25^{-} \mathrm{T}$ cells $\left(\mathrm{CD}^{+} \mathrm{CD}^{+} \mathrm{CD} 25^{-}\right.$, from $\mathrm{CD} 4^{+} \mathrm{CD} 25^{+}$negative selection), and $\mathrm{CD} 4^{+} \mathrm{CD} 25^{+}\left(\mathrm{CD} 3^{+} \mathrm{CD} 4^{+} \mathrm{CD} 25^{+}\right.$ CD127 ${ }^{\text {low }}$, from Treg-positive selection).

\section{In vivo surgery and adoptive transfer}

A series of in vivo experiments were performed by using an excisional cutaneous wound mouse model controlled for contracture, which more closely resembles wound repair by secondary intention. Briefly, mice were anesthetized with 2-3\% isoflurane via inhalation, followed by shaving of dorsal skin and preparation by scrubbing alternately with isopropyl alcohol and betadine. Two full-thickness excisional wounds were created on the dorsum of 8- to 10week-old C57BL/6J or SCID female mice by using a 6-mm punch biopsy (Miltex, Plainsboro, NJ), stented, covered with a sterile adhesive dressing (Tegaderm; 3M, St. Paul, MN), and allowed to heal. Wounds were harvested at day 1, 3, 7, 14, and 30 for analysis. ${ }^{16} n \geq 3$ per time point per condition.

Each mouse in the adoptive transfer experimental groups received $1 \times 10^{6}$ cells of total lymphocytes, $\mathrm{CD} 4^{-}, \mathrm{CD} 4^{+} \mathrm{CD} 25^{-}$, or $\mathrm{CD} 4^{+} \mathrm{CD} 25^{+} \mathrm{T}$ cells isolated from pooled WT mice splenocytes by retro-orbital injection within $6 \mathrm{~h}$ after wounding. Cells were diluted in $100 \mu \mathrm{L}$ of sterile phosphatebuffered saline (PBS) buffer.

\section{Histology and immunohistochemistry}

Tissues were dehydrated, paraffin-embedded, and cut into 5- $\mu \mathrm{M}$ thick sections. Trichrome staining (38016SS2; Leica Biosystems, Buffalo Grove, IL) and Herovici staining (American MasterTech Scientific Laboratory Supplies KTHERPT, Lodi, CA) were performed with a Leica ST5020 Autostainer (Richmond, IL). A standard immunohistochemistry (IHC) protocol was followed for all stains, which were performed on a Dako Autostainer Link 48 and its accompanying software (DakoLink version 4.1, edition 3.1.0.987; Agilent, Santa Clara, CA). Primary antibodies- $\alpha$-smooth muscle actin ( $\alpha$-SMA) for myofibroblasts (ab5694; Abcam, Cambridge, MA), CD45 for pan-leukocytes (ab10558), CD31 for microvasculature (ab28364), and F4/80 for pan-macrophages (ab111101) were detected by EnVision+System-HRP (DAB) kits (Dako North America, Carpinteria, CA).

\section{Immunofluorescence staining}

We adapted the immunofluorescence (IF) staining protocol in this study from Cheng et al. ${ }^{17}$ Briefly, tissues were dehydrated, paraffin-embedded, and cut into $5-\mu \mathrm{M}$ thick sections that were mounted onto slides. Sections were deparaffinized and blocked with $5 \%$ goat serum in PBS for $1 \mathrm{~h}$ at room temperature. Antibodies were diluted in PBS with $1 \%$ bovine serum albumin. Anti-CD3 ${ }^{+}$(Abcam ab16669), anti-CD $4^{+}$(Thermo Fisher MA1-146, Carlsbad, $\mathrm{CA})$, anti-CD8a ${ }^{+}$(eBioscience 45-0081-80), anti-CD25 ${ }^{+}$ (Thermo Fisher MA512680) and anti-FoxP3 ${ }^{+}$ (Thermo Fisher 14-5773-82), anti-rabbit Texas Red (Thermo Fisher T2767), anti-rat Texas Red (Thermo Fisher T-6392), and anti-mouse Alexa Flour 594 (Thermo Fisher A-11032) were used for IF staining.

\section{Wound analysis}

Using hematoxylin and eosin (H\&E)-stained slides, we assessed the neo-epidermis length and granulation tissue area. Neo-epidermis length was measured as the newly generated epidermis from the day 0 epidermis to day 7 neo-epidermis margins on both ends of the healing wounds. Granulation tissue areas were measured by starting from one end of epithelial margin, down to the panniculus carnosus on the same side, then across the wound cleft to the opposite original wound margin, and finally back to the other epithelial margin. Besides the analysis above, $\alpha$-SMA, anti-CD31, anti-CD45, and anti-CD4/ 80 staining were also analyzed with Leica application Suite X version 3.0.4.16319. Image-J 1.50i was used to analyze trichrome and Herovici staining.

\section{Microscopy}

Histology slides were imaged on a Leica DM2000 with Leica application Suite X version 3.0.4.16529. IF slides were imaged using a Leica DMi8 and Leica application Suite X version 3.0.4.16319.

\section{Statistical analysis}

Statistical analysis was performed in Prism software using one-way analysis of variance, followed by Student's $t$-test when appropriate. All quantitative data are expressed as mean \pm standard deviation. $p<0.05$ was considered statistically significant. $N \geq 3$ animals were used in each condition of all our experiments.

\section{RESULTS \\ $\mathrm{CD4}^{+} \mathrm{T}$ lymphocytes are present throughout the wound repair process}

To define the temporal and spatial distribution of $\mathrm{T}$ cells in normal dermal wound healing, we performed IF staining on C57BL/6J WT mouse skin with anti$\mathrm{CD}^{+}{ }^{+}$, anti-CD4 ${ }^{+}$, anti-CD8 ${ }^{+}$, anti-CD25 ${ }^{+}$, and antiFoxP3 $^{+}$antibodies. Figure 1A shows representative images of normal mouse skin at day 0 (unwounded skin) and day 1, 3, 7, 14, and 30 post-wounding, which reveals the presence of $\mathrm{CD}^{+} \mathrm{T}$ cell infiltration at all time points. These results are quantified in Fig. 1B. We found that in unwounded skin, T cells were predominately localized in the epidermis (Fig. 1B, green line). In contrast, $\mathrm{T}$ cells could be observed in the 


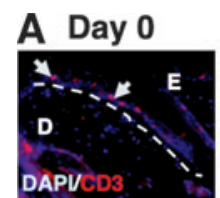

Day 7

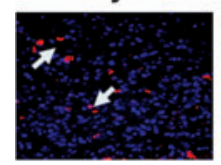

C Day 0
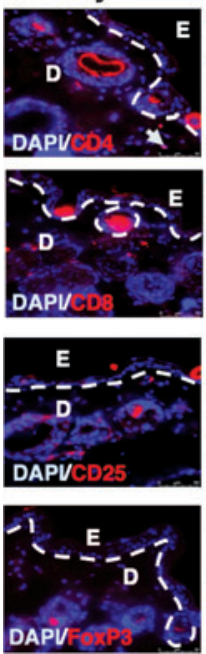

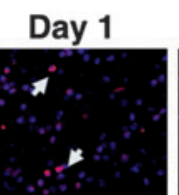

Day 14

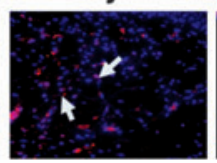

Day 1
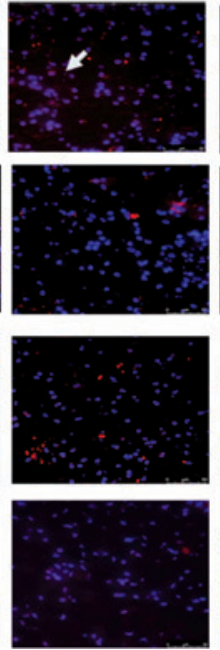

Day 3

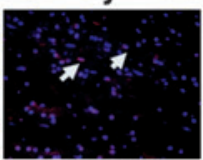

Day 30

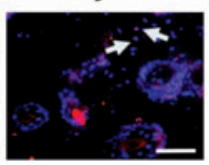

Day 3
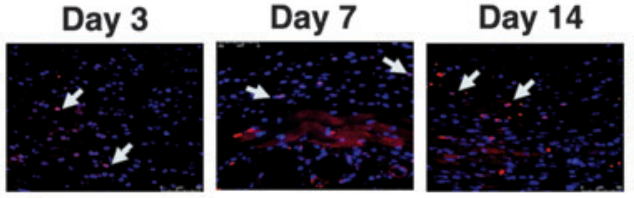

Day 30
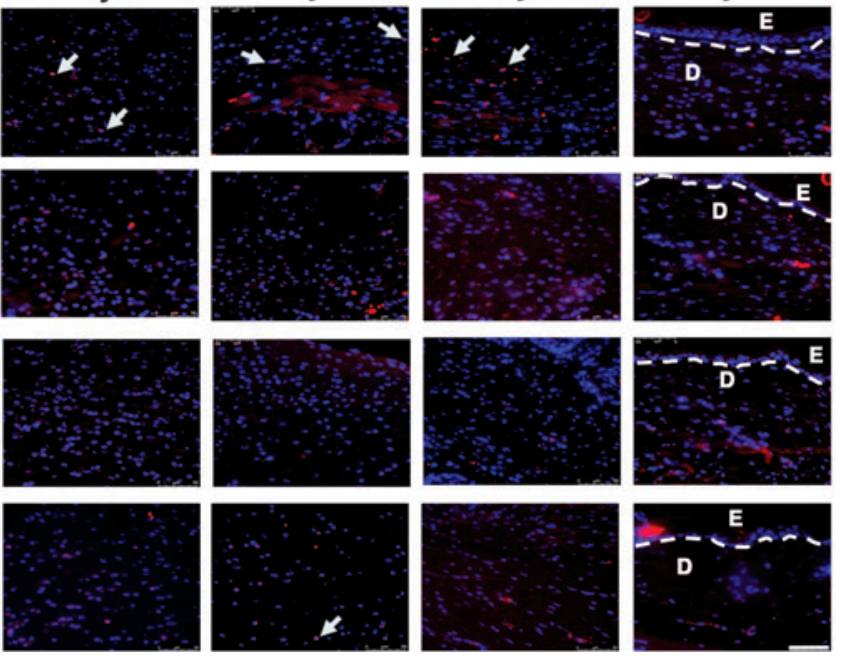
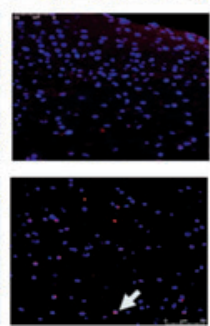

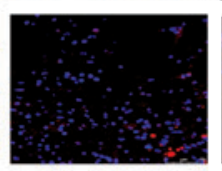

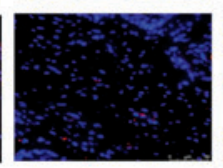
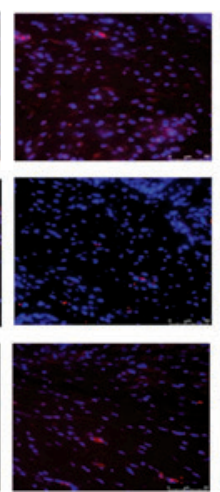

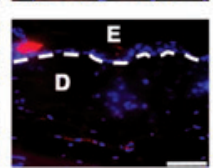

Figure 1. $\mathrm{CD}^{+}$and $\mathrm{CD}^{+} \mathrm{T}$ lymphocytes are present throughout wound repair process up to day 30 post-wounding. (A) Representative images of IF staining of $\mathrm{CD}^{+}$ cells in unwounded skin (day 0 ) and in the wounded area at day 1, 3, 7, 14, and 30 post-wounding. Epidermis (E) and dermis (D) are shown for the day 0 image, and dermis only are shown in the remaining time points. White arrows indicate positively stained cells, and white dotted lines indicate the basement membrane zone. Scale bars: $50 \mu \mathrm{m}$. (B) Quantification of $\mathrm{CD}^{+}$cells per high-power field from images in (A) Green dots/line indicate the average $\mathrm{CD}^{+}$T lymphocytes count in the epidermis per highpower field at the specified time points. Columns indicate the average $\mathrm{CD}^{+}$T lymphocytes count in dermis of the wound area at specified time points. (C) Representative images of IF staining of $\mathrm{CD}^{+}, \mathrm{CD}^{+}, \mathrm{CD}^{\circ} 5^{+}$, and Foxp3 ${ }^{+}$cells in unwounded skin (day 0 ) and in the wounded area at day 1, 3, 7, 14, and 30 post-wounding. Epidermis (E) and dermis (D) are shown for the days 0 and 30 images, and dermis only is shown in the remaining time points. Scale bars: $50 \mu$ m. IF, immunofluorescence.

reticular dermis of wound edges at day 1 and 3 postinjury. T lymphocyte abundance peaked on day 14 post-wounding in the infiltrating wound margins. At day 30 post-wounding, $\mathrm{T}$ cells were significantly reduced and mostly found in the papillary dermis, close to the hair follicle bulges. We then further identified specific T cell subsets present in the wound environment, as shown in Fig. 1C. We observed CD4 ${ }^{+}$and $\mathrm{FoxP}^{+}$, but not $\mathrm{CD}^{+}$or $\mathrm{CD} 25^{+}$, cells in the wound area at different time points. These data suggest that $\mathrm{CD}^{+}$cells are the predominant $\mathrm{T}$ cell lineage in healing wounds.

\section{Lymphocyte deficiency leads to accelerated epithelization but excessive scarring}

To investigate the pathophysiological relevance of $\mathrm{T}$ cells in the injured dermis, we compared day 7 wounds in SCID and WT mice. SCID wounds exhibited quantitatively larger neo-epidermis length (SCID vs. WT: $3.03 \pm 0.41 \mathrm{~mm}$ vs. $1.88 \pm 0.63 \mathrm{~mm}$; $p<0.05$ ) (Fig. 2A, B). Additionally, in comparison to
WT control wounds, SCID mice exhibited significantly larger granulation tissue area, as measured by computer-assisted morphological analysis (SCID vs. WT: $0.63 \pm 0.21 \mathrm{~mm}^{2}$ vs. $0.48 \pm 0.11 \mathrm{~mm}^{2} ; p<0.05$ ) (Fig. 2A, B).

To further assess the impact of an immunocompromised microenvironment on skin wound healing, we compared scar formation between WT and SCID wounds at 7 and 30 days post-wounding via trichrome, Herovici, and IHC analyses. SCID wounds exhibited higher collagen deposition by trichrome staining, more immature collagen (shown in blue) by Herovici staining, and significantly more $\alpha$-SMA-positive myofibroblasts at the wound edges than WT controls (Fig. 2C). These findings were statistically corroborated by computer-assisted morphological analysis (Fig. 2D), suggesting that wound repair undergoes accelerated closure and increased fibrotic scarring in the absence of $\mathrm{T}$ and $\mathrm{B}$ cells. Together, these data indicate a significant impact of lymphocytes on dermal wound healing. 

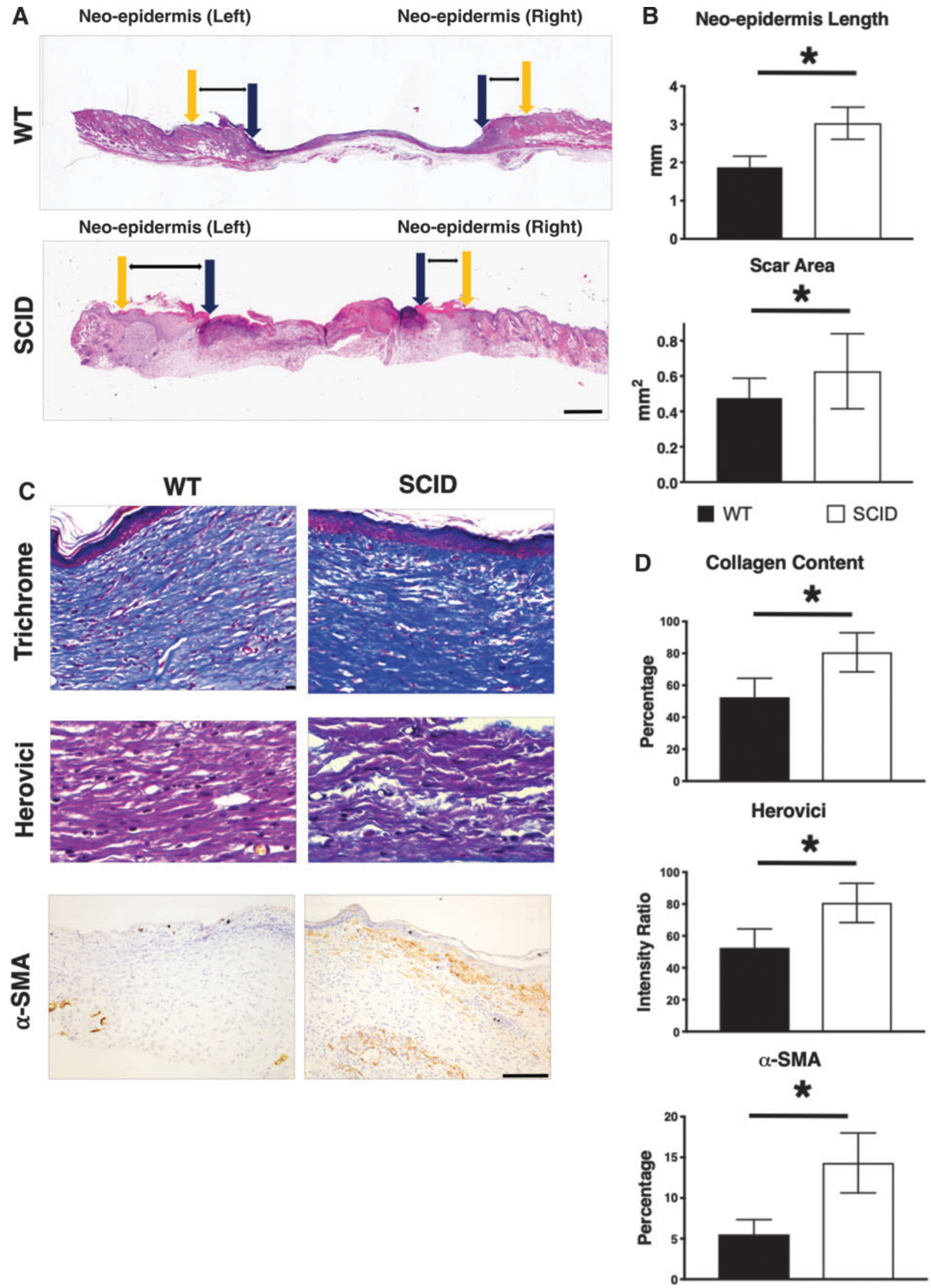

Figure 2. A deficiency of lymphocytes in vivo is associated with accelerated epithelization and fibrosis. (A) Representative images of H\&E staining at day 7 post-wounding in WT and SCID tissue sections. Yellow arrows indicate the original wound edges, and the indigo arrows indicate the neo-epidermis ends (current closed wound edges). The neo-epidermis length is sum of left and right sides epithelial edges. Scale bar: $500 \mu \mathrm{m}$. (B) H\&E image quantification of (A) demonstrating differences in neo-epidermis length and granulation tissue area (for thickness of the wounds). ${ }^{*} p<0.05$. (C) White arrows indicate positively stained cells, and white dotted lines indicate the basement membrane zone. Scale bars: $75 \mu \mathrm{m}$. (D) Quantification of fibrosis burden of images in (C) via collagen content (trichrome), collagen bundle maturation (Herovici), and $\alpha$-SMA as a percentage of total wound area. ${ }^{*} p<0.05 . \alpha$-SMA, $\alpha$-smooth muscle actin; $\mathrm{H} \& \mathrm{E}$, hematoxylin and eosin; IHC, immunohistochemistry; SCID, severe combined immunodeficient; WT, wild type. 


\section{Excessive inflammation and decreased vessel formation alter scid wound healing process}

Next, to investigate potential mechanisms leading to increased dermal scarring, we assessed specific parameters of inflammatory responses in SCID wounds 7 days after wounding. We stained WT and SCID dermal tissue with anti-CD45 and anti-F4/80 antibodies and found that both leukocytes (SCID vs. WT: $34.53 \% \pm 16.57 \%$ vs. $21.27 \% \pm 17.98 \%$; $p<0.05$ ) and macrophages (SCID vs. WT: $8.47 \% \pm$ $2.96 \%$ vs. $5.51 \% \pm 2.15 \%$; $p<0.05)$ were significantly increased in SCID skin wounds compared with WT controls (Fig. 3A, B). These results suggest that, in the absence of $B$ and $T$ cells, the ability to modulate the magnitude of inflammatory responses is lost.

We next investigated whether defective control of inflammatory reactions originating from lymphocyte deficiency impinges upon angiogenesis at day 30 post-wounding. In contrast to WT dermal wounds, SCID sections revealed a marked decrease of CD31 ${ }^{+}$ microvasculature (SCID vs. WT: $4.58 \pm 1.73$ vs. $8.54 \pm 3.21$ counts/high power field; $p<0.05$ ). These data suggest that in the absence of lymphocytes, activation and mobilization of other leukocyte lineages lead to proinflammatory reactions and altered neovascularization (Fig. 3C).

\section{$\mathrm{CD4}^{+} \mathrm{T}$ cells regulate inflammation and reduce matrix accumulation in SCID dermal wound healing}

To determine whether $\mathrm{T}$ cells contribute to dermal wound healing, we adoptively transferred syngeneic $\mathrm{T}$ cell subpopulations to SCID mice within $6 \mathrm{~h}$ after wounding. Briefly, four lymphocyte subset groups - total (T and B lymphocytes), $\mathrm{CD}^{-}\left(\mathrm{CD}^{+}\right.$ $\mathrm{T}$ and $\mathrm{B}$ cells), $\mathrm{CD}^{+}\left(\mathrm{CD}^{+} \mathrm{CD} 4^{+} \mathrm{CD} 25^{-}\right)$, or Treg
$\left(\mathrm{CD}^{+} \mathrm{CD} 4^{+} \mathrm{CD} 25^{+} \mathrm{CD} 127^{\text {low }}\right)$ lymphocytes-from strain-, gender-, and age-matched WT mice were transplanted into SCID mice through retro-orbital injection. Successful lymphocyte transfer was assessed by flow cytometry for CD3, B220, CD4, CD8a, CD25, and CD127 expression on adoptively transferred SCID mice at day 7 and compared with control WT and SCID mice.

As anticipated, the flow cytometry data confirmed that all subpopulations were quantitatively present at day 7 , with biologically equivalent ratios (Fig. 4A). To test the physiological effect of adoptively transferred cells on inflammatory reactions at day 7, we performed IHC to assess the presence or absence $\mathrm{CD} 45^{+}$leukocytes and $\mathrm{F} 4 / 80^{+}$macrophages in the wound microenvironment (Fig. 4B). We observed that in comparison to wounds of untreated SCID mice, the transplant of total lymphocytes, CD4 $4^{-}$lymphocytes, or $\mathrm{CD} 4^{+} \mathrm{T}$ lymphocytes, but not Treg lymphocytes, led to a reduction of leukocyte infiltration. Similarly, macrophage influx was significantly reduced in wounds of SCID mice treated with total lymphocytes and $\mathrm{CD} 4^{+} \mathrm{T}$ lymphocytes. $\mathrm{CD}^{-}$and Treg subpopulations also reduced macrophage infiltration, but to a lesser degree.

We next investigated the effects of adoptively transferred $\mathrm{T}$ cell subpopulations on wound healing outcomes. Transfer of total lymphocytes significantly improved the outcome of wounding in SCID mice with respect to collagen bundle organization and $\alpha$-SMA expression. Similarly, transplant of Treg subsets to wounded SCID mice resulted in significant reduction of $\alpha$-SMA-positive myofibroblasts, but not the collagen content. Finally, transplanting $\mathrm{CD}^{-}$or $\mathrm{CD}^{+}$lymphocytes to SCID mice decreased both myofibroblasts and collagen deposition; however, the level of reduction was
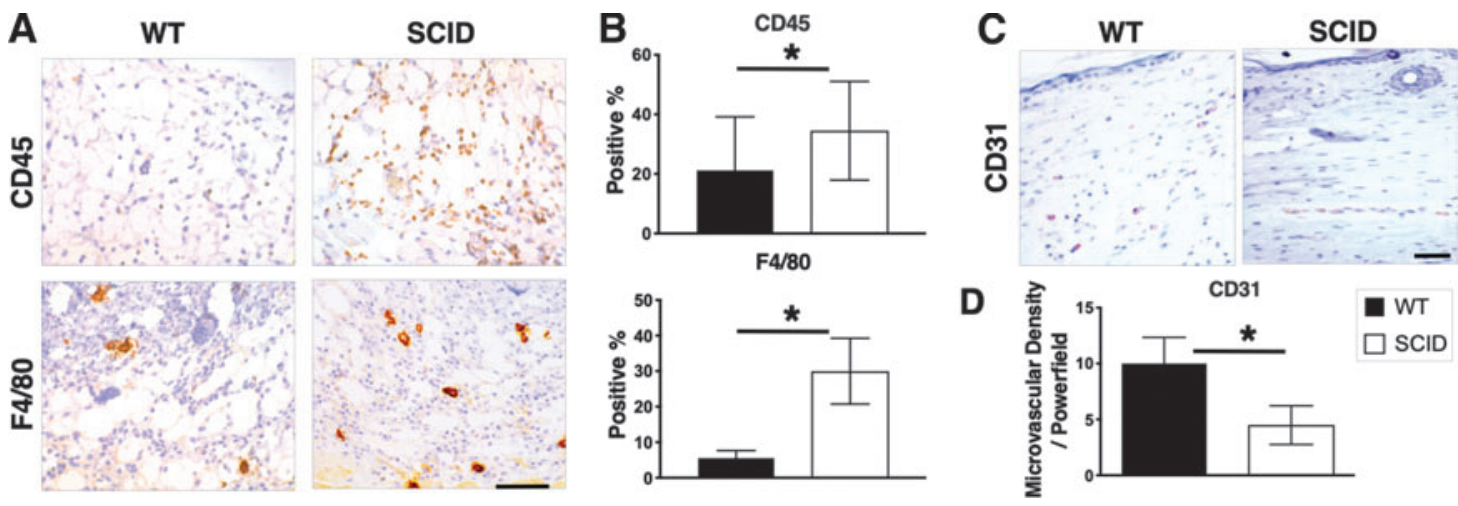

Figure 3. Excessive inflammation and decreased vessel formation prohibit normal wound healing in SCID mice. (A) Representative images of IHC-labeled

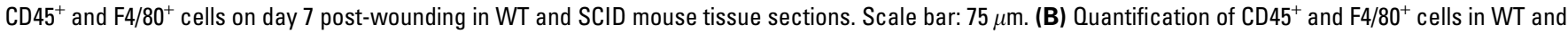
SCID mouse wounds. ${ }^{*} p<0.05$. (C) Representative images of IHC-labeled CD $31^{+}$cells at day 30 post-wounding in WT and SCID mouse tissue sections. Scale bar: $100 \mu \mathrm{m}$. (D) Quantification of CD31 ${ }^{+}$cells in WT and SCID mouse wounds reported as microvascular density per high-power field. ${ }^{*} p<0.05$. 
A

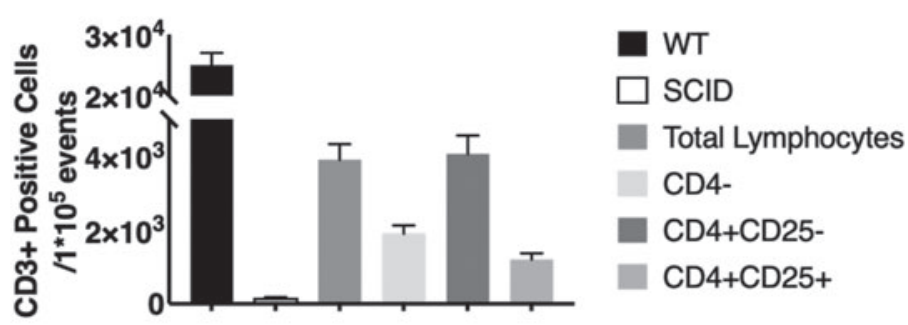

B

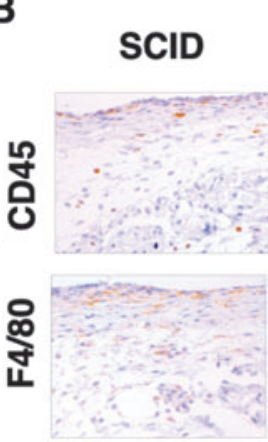

D

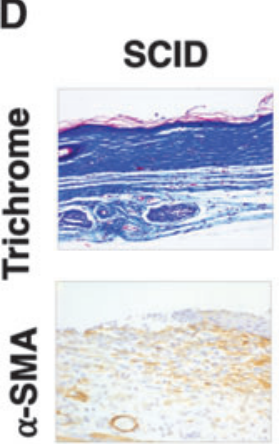

$\mathbf{F}$

ร

SCID
Total Lymphocytes
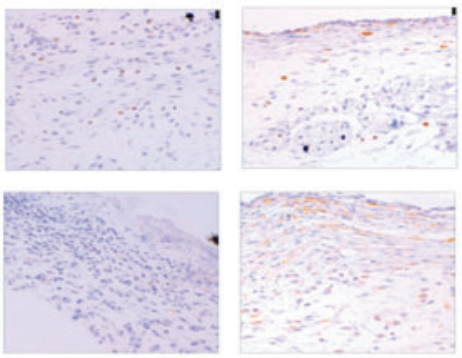

Total Lymphocytes
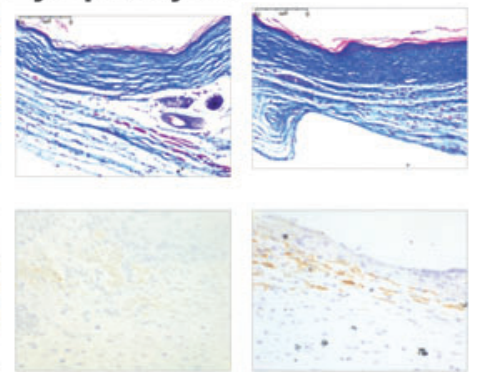

Total

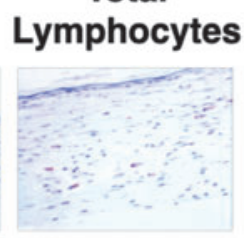

CD4-

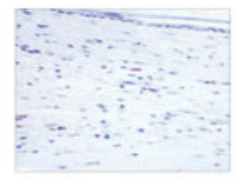

CD4+CD25- CD4+CD25+
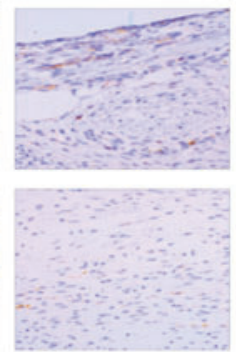

CD4+CD25-
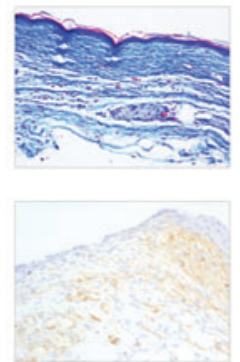

CD4+CD25-

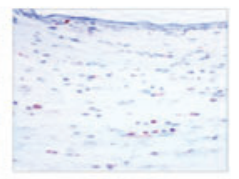

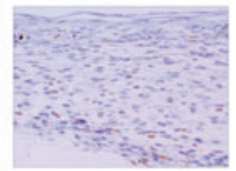

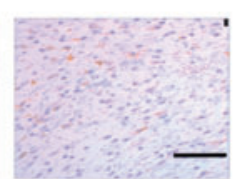

E
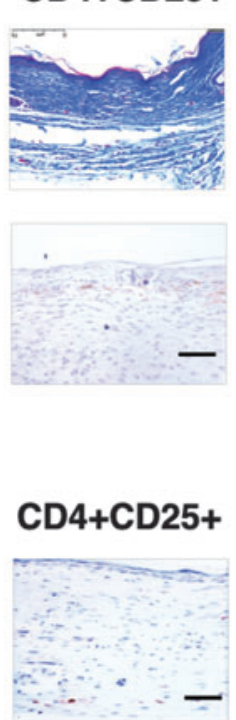

G
C
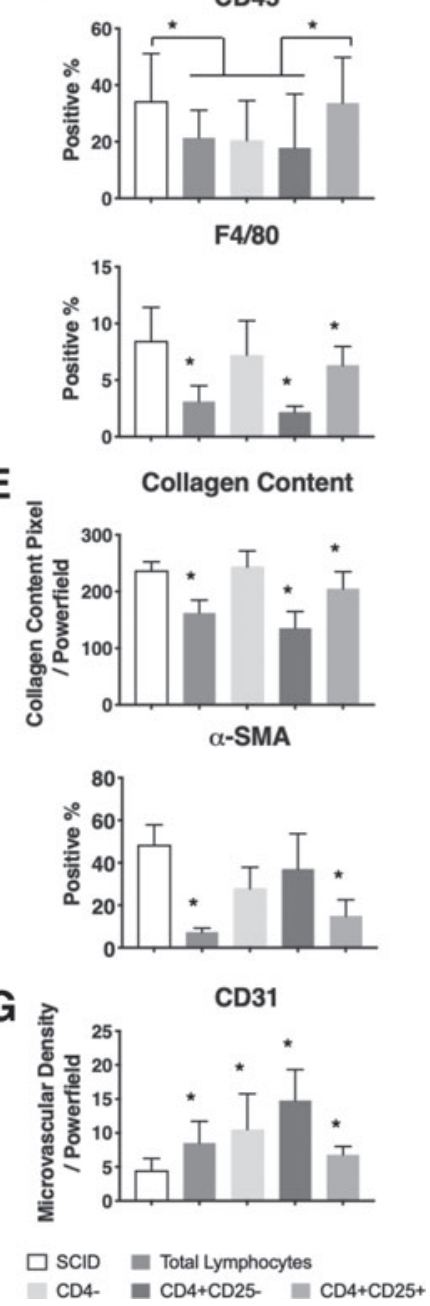

Figure 4. $\mathrm{CD}^{+} \mathrm{T}$ lymphocytes are associated with improved dermal wound healing in SCID mice. (A) Expansion level of CD3 ${ }^{+}$lymphocytes after 7 days postadoptive transfer in each indicated condition through flow cytometry, using WT total lymphocytes as a positive control and untreated SCID lymphocytes as a negative control; $n \geq 3$ per condition. (B) Representative images of IHC-labeled CD45 and F4/80 ${ }^{+}$cells at 7 days post-wounding in untreated SCID mice and SCID mice with adoptive transfer of one of the following subsets: total lymphocytes, $\mathrm{CD} 4^{-}$lymphocytes, $\mathrm{CD} 4^{+} \mathrm{CD} 25^{-}$lymphocytes, or $\mathrm{CD} 4^{+} \mathrm{CD} 25^{+}$lymphocytes. Scale bars: $75 \mu \mathrm{m}$. (C) Quantification of $\mathrm{CD} 45^{+}$and $44 / 80^{+}$cells in each condition from (B). ${ }^{*} p<0.05$ compared with SCID control. (D) Representative images of immunohistochemistry (IHC)-labelled tissue sections of trichrome and Herovici at day 30 post-wounding and alpha-smooth muscle actin ( $\alpha$-SMA) at day 7 postwounding from WT and SCID tissue. Scale bars: $200 \mu \mathrm{m}$. (E) Quantification of collagen content (trichrome) and $\alpha$-SMA levels from D. ${ }^{*} p<0.05$ compared with SCID control. (F) Representative IHC images of CD31 at 30 days post-wounding in control SCID and adoptively transferred SCID with total, $\mathrm{CD}^{-}$, $\mathrm{CD}^{+} \mathrm{CD}^{-} 5^{-}$, or $\mathrm{CD}^{+}{ }^{+} \mathrm{CD} 25^{+}$Iymphocytes. Scale bars: $200 \mu \mathrm{m}$. (G) Quantification of microvascular density from (F). ${ }^{*} p<0.05$ compared with SCID control.

not as prominent as with transfer of total lymphocytes (Fig. 4D, with quantification in Fig. 4E). To further assess the effect of adoptively transferred $\mathrm{T}$ lymphocytes on neovascularization, we examined microvascular formation at day 30 post-lymphocyte transplant in SCID mice by measuring the expres- sion of $\mathrm{CD}^{+} 1^{+}$cells in wounded skin tissue using IHC. We noted that among all the transplanted subsets, $\mathrm{CD} 4^{+} \mathrm{CD} 25^{-}$subsets promoted the highest level of microvascular formation, although total lymphocytes and $\mathrm{CD}^{-}$subsets were also able to promote significant neovascularization (Fig. 4F, G). 
Collectively, our data suggest that SCID mice receiving a transfer of total lymphocytes have significantly improved wound healing outcomes. This occurs in conjunction with reduced inflammation, improved angiogenesis, and attenuated scarring. Of all subsets studied, $\mathrm{CD} 4^{+}$lymphocytes stand out as particularly effective in reducing inflammation and collagen deposition while promoting neovascularization. In contrast, $\mathrm{CD}^{-}{ }^{-}$cells and Treg did not have these effects.

\section{DISCUSSION}

We report that $\mathrm{T}$ cells contribute to reduced scar formation. This work was performed in SCID mice that lack both $\mathrm{B}$ and $\mathrm{T}$ cells and thus provides a direct means to interrogate the impact of lymphocytes on dermal healing. Wounding in SCID mice was associated with faster healing but increased scarring, and transfer of $\mathrm{T}$ cells into SCID mice resulted in reduced scarring. Collectively, our data underscore a restorative role for $\mathrm{T}$ cells in wound healing, including potential underappreciated contributions to ECM remodeling and scar formation.

Notably, $\mathrm{T}$ cells are present in the wound bed at both short- and long-term time points after wounding, as summarized in Fig. 5. We observed T cells in the wounds as early as day 1 , consistent with the finding that $\mathrm{T}$ cells potentially modulate inflammation, promote angiogenesis, and define scarring outcomes. ${ }^{11,12}$

Our data indicate that without functional $\mathrm{T}$ and $\mathrm{B}$ lymphocytes, skin wound closure is accelerated, in- flammation is increased, scar formation is exacerbated, and angiogenesis is decreased. During wound healing, both innate and adaptive immune systems are required for an effective repair process. Since SCID mice lack B and T cells, the increased macrophage infiltration in skin wounds suggests that macrophages may compensate for the altered inflammatory response. Consistent with our observation of increased granulation tissue in SCID wounds, a recent study by Sinha et al. showed direct myeloidto-fibroblast-like cell conversion within the granulation tissue of injury sites in mice. ${ }^{18}$

As we examined the impact of various lymphocyte subsets on our wound healing model using gain-of-function transfer experiments, we concluded that $\mathrm{CD}^{-}{ }^{-}$cells (B cells $+\mathrm{CD}^{+} \mathrm{T}$ cells) have the least effect in reducing scarring outcomes in comparison to total lymphocyte-transplanted SCID mice, although it is able to improve angiogenesis outcomes. To the best of our knowledge, these collective data provide the first evidence that $\mathrm{CD}^{+} \mathrm{T}$ lymphocytes, but not Tregs, play a previously underappreciated regulatory role to reduce scar formation and increase angiogenesis during dermal wound healing.

We chose the C57BL/6J genetic background based on our extensive experience with the model and its utility in concert with the extensively studied T, B, and NK cell-deficient B6.CB17Prkdc ${ }^{\text {scid }} /$ SzJ strain. ${ }^{19-25}$ Additionally, the SCID model is amenable to xenografts, ${ }^{26,27}$ allowing us to perform multiple variations of the adoptive
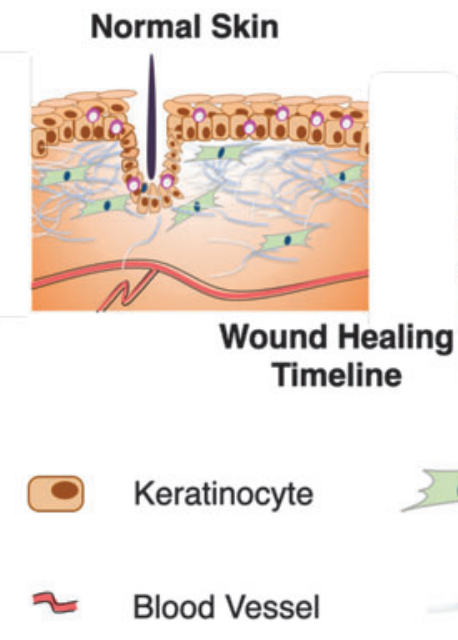

Inflammation

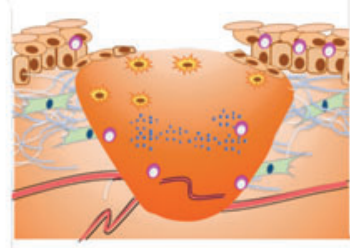

1

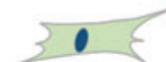

Fibroblast
3
Proliferation

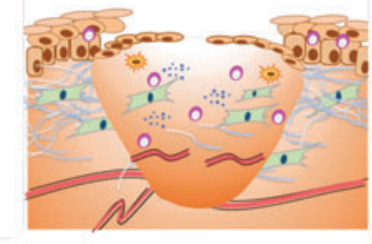

7
14

T cells

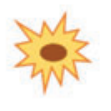

Macrophage

30

Maturation

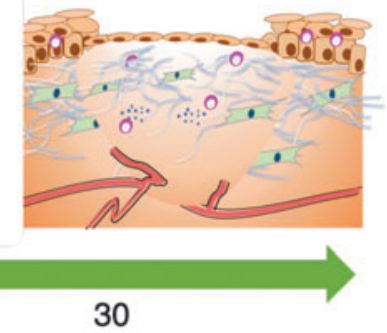

Cytokine

Hair

Figure 5. Schematic illustration of the timeline of T cells involved in wound healing. This schematic illustration reflects what we observed in C57BL/6J WT mouse wound healing at different time points. At all stages of wound healing, we observed T cells in various parts of the skin or wounds, indicating their important role in wound healing. Initially, we observed T cells in only the epidermis of the normal skin. After wounding, a small population of $T$ cells was observed in the deep dermis of the wound edge during the inflammation phase, while macrophages were observed in the upper dermis. More $T$ cells were observed during the proliferation phase, and they were located at the edge and center of the wound dermis. Although we did not observe any $T$ cells in the newly formed epidermis at day 30 post-wounding, they can be seen in the upper dermis or close to the newly formed epidermis. 
transfer to comprehensively investigate how distinct $\mathrm{T}$ lymphocyte subsets contribute to dermal wound healing. An alternative $\mathrm{T}$ lymphocytedeficient mouse model, the NU/J mouse, has been studied in wound healing; however, it has a different wound healing phenotype and skin physiology from the background C57BL/6J mouse; thus, the conclusions from that study are impossible to deconvolve from the strain choice. ${ }^{10}$

Intriguingly, Chen et al. performed comparative studies between WT and immunocompromised mice and reported that $\mathrm{CD}^{+}$or $\mathrm{CD}^{+} \mathrm{T}$ celldeficient mice showed no significant differences in the wound healing process. ${ }^{28}$ However, their data also indicated that $\mathrm{CD}^{+}$or $\mathrm{CD}^{+} \mathrm{T}$ cell deficiency was compensated for by upregulation of other immune cells. ${ }^{28}$ Our results show that although adoptive transfer cannot completely restore normal phenotype, transfer of total lymphocytes to SCID mice achieves the best wound healing outcomes in terms of inflammatory profiles and scarring phenotypes. In terms of specific $\mathrm{T}$ lymphocyte transplant results, $\mathrm{CD}^{+}$reconstituted mice showed the least scarring, followed by Treg, whereas $\mathrm{CD}^{-}\left(\mathrm{CD}^{+} \mathrm{T}\right.$ cells and B cells) cells had no impact on scarring in transplanted SCID mice when compared with nontransplanted SCID mice. In support of our findings, previous studies have shown that $\mathrm{CD} 4^{+} \mathrm{T}$ cells contribute to the repair of injured organs, including the heart, ${ }^{29}$ kidney, ${ }^{30}$ and lung, ${ }^{31}$ by either secreting or inducing other effector cells to produce cytokines, chemokines, and growth factors to regulate tissue repair and scarring. ${ }^{32}$

We reported that Tregs help maintain immunologic tolerance in healing tissues ${ }^{5}$ and respond to ECM microenvironment signaling, which could promote the expansion of Tregs from precursors to modulate inflammatory reactions. ${ }^{33}$ Among all T lymphocyte subpopulations, the Treg subset is known for its ability to dampen overaggressive responses and is necessary for tissue neovascularization, ${ }^{34-36}$ although its role in dermal wound healing could vary depending on the host. For example, Tregs have been shown to facilitate wound repair in a FoxP3 $^{+}$Treg conditionalknockout mouse model, but detrimental to skin wound healing in a diabetic mouse model with a genetically engineered CCR4 deficiency. ${ }^{37}$ In our adoptive transfer experiments, we demonstrated that SCID mice receiving Treg cells had reduced scarring compared with other T cell subsets, with the exception of the $\mathrm{CD}^{+}$subset. Although adoptive transfer of Tregs did increase angiogenesis, the improvement was lowest among all treatment groups. We would posit that limited systemic IL-2 levels resulting from limited microbial stimulation may have failed to fully activate $\mathrm{FoxP}^{+}{ }^{\text {Treg. }}{ }^{38}$ In the future, we could address this question by providing low-dose IL-2 to enhance Treg activity or suppress $\mathrm{T}$ and $\mathrm{B}$ cells sensitivity to IL-2 by rapamycin or metformin.

In sum, to fully understand the spatial and temporal contribution of $\mathrm{T}$ cells to physiological dermal wound healing, it will be necessary to elucidate how concerted cellular interactions between resident and recruited subsets are governed and how they respond to dermal injury cues to modulate wound healing. We are also aware that most immunodeficient mouse models cannot completely recapitulate human immune responses. Furthermore, the distinct differences in living environment between experimental rodents and humans are a significant factor in wound healing outcomes, especially considering the limited versus continuous exogenous immune challenges faced by rodent models versus humans, respectively. ${ }^{39}$ Future work will be needed to investigate the origin, identity, and function of the specific $\mathrm{T}$ lymphocyte mediators we observed in the wound bed at various time points, especially at the early stages of wound repair. To conclude, our understanding of how $\mathrm{T}$ lymphocytes and respective subsets coordinate the responses to dermal injury to provide a given tissue repair outcome could shed light on how to mitigate scar formation in dermal wounds.

\section{INNOVATION}

Although significant progress has been achieved in understanding the transition from inflammation to scarring in dermal wound healing, a significant gap in knowledge remains regarding the role of lymphocytes in regulating the direction of wound repair-with or without scaring. Our data unveil an underappreciated function of $\mathrm{T}$ lymphocyte subsets to attenuate the degree of inflammation and promote relevant neovascularization, thereby reducing the risk of dermal scarring. The time course of $\mathrm{T}$ lymphocyte infiltration into the wound is a new finding, and the differential T lymphocyte transplant approach to specifically query subsetspecific contribution(s) to the pathogenesis of scarring in SCID mice is intuitive. These studies are geared to yield new clues to design personalized precision immunotherapies.

\section{ACKNOWLEDGMENTS AND FUNDING SOURCES}

We thank the editorial support of Drs. Monica Fahrenholtz and Hector Martinez-Valdez from the 
Office of Surgical Research Administration and Ms. Jill Tuley from the Division of Pediatric Surgery at Texas Children's Hospital (TCH). We are also grateful for the generous support and immunologic perspective from Dr. Katie Matatall, Dr. Katherine King, and Ms. Claudine Salire Kadmon. This work was supported by NIH 1R01GM111808 and a generous gift from Amie and Jeff Springmeyer to support research in regenerative medicine (principal investigator [PI]: S.G.K.); Dr. Ralph and Marian Falk Medical Research Trust Bank of America, N.A., Trustee, R01AI12 492093, R01DK114174-01A1, R01DK096087-07, U01 AI101984, and R21AI133370 (PI: P.L.B.); NIH R0 GM110482 and the E. Richard Stiehm Endowment that supports research in immunology (PI: M.J.B.).

\section{AUTHOR DISCLOSURE AND GHOSTWRITING}

The article was written by the authors and ghostwriting services were not used. Editorial support provided by Drs. Hector Martinez-Valdez and Monica Fahrenholtz at the Office of Surgical Research Administration at TCH.

\section{ABOUT THE AUTHORS}

Xinyi Wang, PhD, is a Postdoctoral Fellow in the Laboratory of Regenerative Tissue Repair (LRTR), who focuses on inflammation and tissue fibrosis. Swathi Balaji, PhD, is an Assistant Professor in the Department of Surgery at the
- $\mathrm{CD}^{+}$Iymphocytes are present in the wound at day 3, peak at day 14 and persist until day 30, suggesting a significant $T$ lymphocyte role in dermal wound healing and scarring responses.

- In the B and T lymphocyte-deficient SCID mouse, wound repair results in accelerated epithelial wound closure, increased inflammation, decreased angiogenesis, and exacerbated scar formation.

- $\mathrm{CD}^{+}{ }^{+} \mathrm{T}$ lymphocytes may represent the key lymphocyte population that regulates the responses to wound injury and repair. There is a balance between inflammation angiogenesis directed by $T$ lymphocytes that may be a part of the mechanisms that account for tissue repair and scar formation.
Baylor College of Medicine who focuses on the exosome regulation of tissue fibrosis. Emily $\mathbf{H}$. Steen, MD, is a surgical resident at the Baylor College of Medicine, who is completing a 2-year postdoctoral fellowship. Hui Li, PhD, is a research associate in the LRTR. Qi Miao, PhD, is a postdoctoral fellow in the LRTR. Meredith M. Rae, BS, and Alexander J. Blum, BS, are former predoctoral fellows in the LRTR and are now in medical school at UT Southwestern and Johns Hopkins Medical School, respectively. Manish J. Butte, MD, PhD, is the Chief of the Division of Allergy and Immunology at the David Geffen School of Medicine at University of California, Los Angeles. Paul L. Bollyky, MD, PhD, is an Assistant Professor in the Division of Infectious Disease at Stanford University. Sundeep G. Keswani, MD, is the Director of Surgical Research at TCH and is the PI for the LRTR.

\section{REFERENCES}

1. Zhang C, Li L, Feng K, Fan D, Xue W, Lu J. "Repair" Treg cells in tissue injury. Cell Physiol Biochem 2017:43:2155-2169.

2. Chazaud B. Macrophages: supportive cells for tissue repair and regeneration. Immunobiology 2014;219:172-178.

3. Keyes BE, Liu S, Asare A, et al. Impaired epidermal to dendritic $T$ cell signaling slows wound repair in aged skin. Cell 2016;167:1323.e13141338.e1314.

4. Gurtner GC, Werner S, Barrandon Y, Longaker MT Wound repair and regeneration. Nature 2008;453: 314-321

5. Bollyky PL, Falk BA, Long SA, et al. CD44 costimulation promotes FoxP3 ${ }^{+}$regulatory $\mathrm{T}$ cell persistence and function via production of IL-2, IL-10, and TGF-beta. J Immunol 2009;183:2232-2241.

6. Kuipers HF, Rieck M, Gurevich I, et al. Hyaluronan synthesis is necessary for autoreactive T-cell trafficking, activation, and Th1 polarization. Proc Natl Acad Sci U S A 2016;113:1339-1344.

7. Sîrbulescu RF, Boehm CK, Soon E, et al. Mature B cells accelerate wound healing after acute and chronic diabetic skin lesions. Wound Repair Regen 2017;25:774-791

8. Gawronska-Kozak B, Bogacki M, Rim JS, Monroe WT, Manuel JA. Scarless skin repair in immunodeficient mice. Wound Repair Regen 2006;14: 265-276

9. Vacchelli E, Semeraro M, Enot DP, et al. Negative prognostic impact of regulatory T cell infiltration in surgically resected esophageal cancer postradiochemotherapy. Oncotarget 2015;6:2084020850.

10. Wong VW, Paterno J, Sorkin M, et al. Mechanical force prolongs acute inflammation via T-celldependent pathways during scar formation. FASEB J 2011;25:4498-4510.
11. Haertel E, Joshi N, Hiebert P, Kopf M, Werner S. Regulatory $T$ cells are required for normal and activin-promoted wound repair in mice. Eur $J$ Immunol 2018;48:1001-1013.

12. Guo S, Dipietro LA. Factors affecting wound healing. J Dent Res 2010;89:219-229.

13. Lei H, Schmidt-Bleek K, Dienelt A, Reinke P, Volk HD. Regulatory T cell-mediated anti-inflammatory effects promote successful tissue repair in both indirect and direct manners. Front Pharmacol 2015;6:184

14. Ruppert SM, Falk BA, Long SA, Bollyky PL. Regulatory $T$ cells resist cyclosporine-induced cell death via CD44-mediated signaling pathways. Int J Cell Biol 2015;2015:614297.

15. Matatall KA, Shen CC, Challen GA, King KY. Type II interferon promotes differentiation of myeloidbiased hematopoietic stem cells. Stem Cells 2014; 32:3023-3030. 
16. Balaji S, Wang X, King A, et al. Interleukin-10mediated regenerative postnatal tissue repair is dependent on regulation of hyaluronan metabolism via fibroblast-specific STAT3 signaling. FASEB J 2017;31:868-881.

17. Cheng CF, Sahu D, Tsen F, et al. A fragment of secreted Hsp90alpha carries properties that enable it to accelerate effectively both acute and diabetic wound healing in mice. J Clin Invest 2011;121:4348-4361.

18. Sinha M, Sen CK, Singh K, et al. Direct conversion of injury-site myeloid cells to fibroblast-like cells of granulation tissue. Nat Commun 2018;9:936.

19. Shultz LD, Goodwin N, Ishikawa F, Hosur V, Lyons $\mathrm{BL}$, Greiner DL. Human cancer growth and therapy in immunodeficient mouse models. Cold Spring Harb Protoc 2014;2014:694-708.

20. Walsh NC, Kenney LL, Jangalwe S, et al. Humanized mouse models of clinical disease. Annu Rev Pathol 2017;12:187-215.

21. Bellinghausen I, Saloga J. Analysis of allergic immune responses in humanized mice. Cell Immunol 2016;308:7-12.

22. Allweiss L, Dandri M. Experimental in vitro and in vivo models for the study of human hepatitis $B$ virus infection. J Hepatol 2016;64(1 Suppl):S17-S31.

23. Staal FJ, Wiekmeijer AS, Brugman MH, PikeOverzet K. The functional relationship between hematopoietic stem cells and developing T lymphocytes. Ann N Y Acad Sci 2016;1370:36-44.

24. Bruttel VS, Wischhusen J. Cancer stem cell immunology: key to understanding tumorigenesis and tumor immune escape? Front Immunol 2014;5:360.

25. Bosma M, Schuler W, Bosma G. The SCID mouse mutant. Curr Top Microbiol Immunol 1988;137: 197-202.
26. Hudson WA, Li Q, Le C, Kersey JH. Xenotransplantation of human lymphoid malignancies is optimized in mice with multiple immunologic defects. Leukemia 1998;12:2029-2033.

27. Paton-Hough J, Chantry AD, Lawson MA. A review of current murine models of multiple myeloma used to assess the efficacy of therapeutic agents on tumour growth and bone disease. Bone 2015;77:57-68.

28. Chen L, Mehta ND, Zhao Y, DiPietro LA. Absence of CD4 or CD8 lymphocytes changes infiltration of inflammatory cells and profiles of cytokine expression in skin wounds, but does not impair healing. Exp Dermatol 2014;23:189-194.

29. Hofmann U, Beyersdorf N, Weirather J, et al. Activation of $\mathrm{CD}^{+} \mathrm{T}$ lymphocytes improves wound healing and survival after experimental myocardial infarction in mice. Circulation 2012;125:1652-1663.

30. Liu L, Kou P, Zeng Q, et al. CD4 ${ }^{+}$T lymphocytes, especially Th2 cells, contribute to the progress of renal fibrosis. Am J Nephrol 2012;36:386-396.

31. Sun J, Madan R, Karp CL, Braciale TJ. Effector T cells control lung inflammation during acute influenza virus infection by producing IL-10. Nat Med 2009;15:277-284.

32. Wynn TA. Cellular and molecular mechanisms of fibrosis. J Pathol 2008;214:199-210.

33. Bollyky PL, Wu RP, Falk BA, et al. ECM components guide IL-10 producing regulatory T-cell (TR1) induction from effector memory T-cell precursors. Proc Natl Acad Sci U S A 2011;108:7938-7943.

34. Nosbaum A, Prevel N, Truong HA, et al. Cutting edge: regulatory $\mathrm{T}$ cells facilitate cutaneous wound healing. J Immunol 2016;196:2010-2014.

35. Bansal SS, Ismahil MA, Goel M, et al. Dysfunctional and proinflammatory regulatory T-lymphocytes are essential for adverse cardiac remodeling in ischemic cardiomyopathy. Circulation 2019;139:206-221.

36. Bollyky PL, Falk BA, Wu RP, Buckner JH, Wight TN, Nepom GT. Intact extracellular matrix and the maintenance of immune tolerance: high molecular weight hyaluronan promotes persistence of induced $\mathrm{CD}^{+} \mathrm{CD}^{2} 5^{+}$regulatory T cells. J Leukoc Biol 2009;86:567-572.

37. Barros JF, Waclawiak I, Pecli C, et al. Role of chemokine receptor CCR4 and regulatory $T$ cells in wound healing of diabetic mice. J Invest Dermatol 2018;139: $1161-1170$.

38. Long SA, Cerosaletti K, Bollyky PL, et al. Defects in IL-2R signaling contribute to diminished maintenance of FOXP3 expression in CD4(+)CD25(+) regulatory T-cells of type 1 diabetic subjects. Diabetes 2010;59:407-415.

39. Tao L, Reese TA. Making mouse models that reflect human immune responses. Trends Immunol 2017;38:181-193.

\section{ABBREVIATIONS AND ACRONYMS}

$\alpha$-SMA $=\alpha$-smooth muscle actin

$\mathrm{ECM}=$ extracellular matrix

$\mathrm{IF}=$ immunofluorescence

$\mathrm{IHC}=$ immunohistochemistry

LRTR = Laboratory of Regenerative Tissue Repair

PBS $=$ phosphate-buffered saline

$\mathrm{PI}=$ principal investigator

SCID $=$ severe combined immunodeficient

$\mathrm{TCH}=$ Texas Children's Hospital

Treg cell $=$ regulatory $\mathrm{T}$ cell

$\mathrm{WT}=$ wild type 